\title{
HAPPEX: Strange Quarks in the Proton and ${ }^{4} \mathrm{He}$
}

\author{
Robert Michaels
}

\author{
Thomas Jefferson National Accelerator Facility
}

\begin{abstract}
HAPPEX results from our 2005 run for the parity-violating asymmetry $A_{\mathrm{PV}}$ in elastic scattering of $3 \mathrm{GeV}$ electrons off hydrogen and ${ }^{4} \mathrm{He}$ targets at $\left\langle\theta_{l a b}\right\rangle \approx 6.0^{\circ}$ are presented. The ${ }^{4} \mathrm{He}$ result is $A_{\mathrm{PV}}=(+6.40 \pm 0.23$ (stat) \pm 0.12 (syst) $) \times 10^{-6}$. The hydrogen result is $A_{\mathrm{PV}}=$ $(-1.58 \pm 0.12$ (stat) \pm 0.04 (syst) $) \times 10^{-6}$. The asymmetry for hydrogen is a linear combination of $G_{E}^{s}$ and $G_{M}^{s}$, the strange quark contributions to the electric and magnetic form factors of the nucleon, respectively, and that for ${ }^{4} \mathrm{He}$ is a function of $G_{E}^{s}$ alone. The measurements imply $G_{E}^{s}+$ $0.09 G_{M}^{s}=0.007 \pm 0.011 \pm 0.006$ at $\left\langle Q^{2}\right\rangle=0.109 \mathrm{GeV}^{2}$ and $G_{E}^{s}=0.002 \pm 0.014 \pm 0.007$ at $\left\langle Q^{2}\right\rangle=0.077 \mathrm{GeV}^{2}$.
\end{abstract}

Keywords: Elastic Electron Scattering, Parity Violation, Nucleon Structure, Strangeness in Nucleon

PACS: $25.30 . \mathrm{Bf}, 13.60 . \mathrm{Fz}, 11.30 . \mathrm{Er}, 13.40 . \mathrm{Gp}, 14.20 . \mathrm{Dh}$

\section{JLAB COPYRIGHT}

Notice: Authored by The Southeastern Universities Research Association, Inc. under U.S. DOE Contract No. DE-AC05-84150. The U.S. Government retains a non-ex clusive, paid-up, irrevocable, world-wide license to publish or reproduce this manuscript for U.S. Government purposes.

\section{INTRODUCTION}

The role of strange quarks in the structure of nucleon, i.e. its mass, spin, momentum, and electromagnetic form factors, has recently been a topic of great interest. A practical way to measure the strange vector matrix elements is by measuring the electroweak asymmetry in polarized electron scattering $[1,2,3,4,5,6,7]$. The asymmetry is $A=$ $\left(\sigma_{R}-\sigma_{L}\right) /\left(\sigma_{R}+\sigma_{L}\right)$ where $\sigma_{R(L)}$ is the differential cross section for elastic scattering of $\operatorname{right}(R)$ and left $(L)$ handed longitudinally polarized electrons from protons. We report here on the 2005 HAPPEX run in Hall A at the Thomas Jefferson National Accelerator Facility (Jefferson Lab). The kinematics were $E_{\text {beam }} \sim 3 \mathrm{GeV}, \theta_{\text {lab }} \sim 6^{\circ}$ and $Q^{2} \sim 0.1$ $\mathrm{GeV}^{2}$.

Electromagnetic scattering at a given kinematics can measure only two linear combinations of the Sachs form factors. For example, the proton form factors in terms of $u, d$, and $s$ quark form factors are:

$$
G_{E, M}^{\gamma p}=\frac{2}{3} G_{E, M}^{u}-\frac{1}{3} G_{E, M}^{d}-\frac{1}{3} G_{E, M}^{S}
$$


where $G_{E, M}^{f}$ is the electric $(E)$ or magnetic $(M)$ form factor for quark flavor $f$. Here it is assumed that the quark flavors $u, d$, and $s$ contribute and the goal is to determine $G_{E, M}^{S}$, the strangeness form factors.

Charge symmetry between the proton $p$ and the neutron $n$ is normally assumed, so that for the quark form factors

$$
G_{p}^{u}=G_{n}^{d} ; \quad G_{p}^{d}=G_{n}^{u} ; \quad G_{p}^{s}=G_{n}^{s}
$$

where the subscripts $p$ and $n$ are for the proton and the neutron, respectively. Note, charge asymmetry may be broken at the level of our experimental error [8].

Since there are too many quark form factors for the given number of nucleon electromagnetic form factors, one needs additional information to determine whether or not there is a contribution from the strangeness form factors $G_{E, M}^{S}$. This is provided by parity violation because the $Z^{0}$ of the weak interaction accesses the same flavor structure with different coupling constants given by the Standard Model. For a proton:

$$
\begin{aligned}
G_{E, M}^{Z p}= & \left(\frac{1}{4}-\frac{2}{3} \sin ^{2} \theta_{W}\right) G_{E, M}^{u}+ \\
& \left(-\frac{1}{4}+\frac{1}{3} \sin ^{2} \theta_{W}\right) \times\left[G_{E, M}^{d}+G_{E, M}^{s}\right]
\end{aligned}
$$

Parity violation in elastic scattering from the ${ }^{4} \mathrm{He}$ nucleus is directly sensitive to the $G_{E}^{s}$. Neglecting well-known radiative corrections the asymmetry is

$$
A_{\mathrm{PV}}=A_{0} \tau\left\{4 \sin ^{2} \theta_{W}+\frac{2 G_{E}^{s}}{G_{E}^{p}+G_{E}^{n}}\right\}
$$

where

$$
A_{0}=\frac{G_{F} M_{P}^{2}}{\sqrt{2} \pi \alpha}=316.7 \mathrm{ppm}
$$

Thus by combining measurements from the proton and from helium, we can separate $G_{E}^{s}$ and $G_{M}^{s}$. The interpretation of the experiments is quite clean, requiring only the assumptions of charge symmetry in the proton and that at our low $Q^{2}$ nuclear effects in ${ }^{4} \mathrm{He}$ are under sufficient control theoretically.

The most recent HAPPEX measurements, presented here, run at very low $Q^{2}$ where one is sensitive mainly to the static moments $\rho_{s}$ and $\mu_{s}$, the strangeness radius and magnetic moment. Most theoretical models focus on predictions of these static moments at $Q^{2}=0$, while only a subset of the models attempt to predict the form factors at higher $Q^{2}$ (for reviews see $[4,5,6,7]$ ). The problem is one of non-perturbative QCD dynamics and is very challenging.

\section{THE HAPPEX EXPERIMENT}

The work presented here has been submitted for publication [9], and the experimental technique was described in detail in $[10,11,12]$. 
The experimental setup uses two identical $3.7 \mathrm{msr}$ spectrometer systems consisting of the Hall A septum magnets plus HRS spectrometers to focus elastically scattered electrons onto total-absorption detectors in their focal planes. A $100 \mu \mathrm{A}, 85 \%$ polarized beam with a $30 \mathrm{~Hz}$ helicity reversal scatter from the targets. Cerenkov light from each detector was collected by a photomultiplier tube, integrated over the duration of each helicity window and digitized by analog to digital converters (ADCs). The HRS pair has sufficient momentum resolution to spatially separate the elastic electrons from inelastic electrons. The amount of background was measured in separate calibration runs in counting mode at low rate using drift chambers. Low rate counting measurements were also done to measure $Q^{2}$. Polarization is measured every few days by a Møller polarimeter and monitored continuously with a Compton polarimeter.

HAPPEX has made tremendous progress in controlling helicity-correlated systematics, achieving helicity correlated position differences which average to $\sim 1$ nanometer during the run. This accomplishment is due to a long-term effort to improve the optics setup at the polarized source as well as the tune of the accelerator. A GaAs photocathode was optically pumped by circularly polarized laser light to produce polarized electrons, with the ability to rapidly and randomly flip the sign of the electron beam polarization. Most of the work to control systematic errors is done at the polarized source. The helicity-correlated intensity asymmetry was maintained to be less than $1 \mathrm{ppm}$ by an active feedback loop. We have a well-developed model for reducing and controlling the laser systematics.

The right-left helicity asymmetry in the integrated detector response, normalized to the beam intensity, was computed for each pair to form the raw asymmetry $A_{\text {raw }}$. The dependence of $A_{\text {raw }}$ on fluctuations in the five correlated beam parameter differences $\Delta x_{i}$ is quantified as $A_{\text {beam }}=\sum c_{i} \Delta x_{i}$, where the coefficients $c_{i}$ quantify the $A_{\text {raw }}$ beam parameter sensitivity. The electroweak physics of the signal and backgrounds is contained in $A_{\text {corr }}=A_{\text {raw }}-A_{\text {beam }}$. The $A_{\text {corr }}$ window-pair distributions for the two complete data samples was Gaussian over more than 4 orders of magnitude, indicating that the main source of noise was counting statistics.

Due to the excellent control of beam parameter differences $\Delta x_{i}, A_{\text {corr }}-A_{\text {raw }}$ values are of the order of, or much smaller than, the corresponding statistical errors. Under $\lambda / 2$ reversal, the absolute values of $A_{\text {corr }}$ are consistent within statistical errors. The reduced $\chi^{2}$ for $A_{\text {corr }}$ "slug" averages is close to one in every case, indicating that any residual beam-related systematic effects were small and randomized over the time

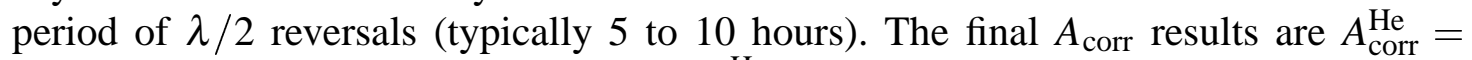
$+5.25 \pm 0.19$ (stat) \pm 0.05 (syst) ppm and $A_{\text {corr }}^{\mathrm{H}}=-1.42 \pm 0.11$ (stat) \pm 0.02 (syst) ppm.

The physics asymmetry $A_{\text {phys }}$ is formed from $A_{\text {corr }}$,

$$
A_{\mathrm{phys}}=\frac{K}{P_{\mathrm{b}}} \frac{A_{\mathrm{corr}}-P_{\mathrm{b}} \sum_{i} A_{i} f_{i}}{1-\sum_{i} f_{i}}
$$

with corrections for the beam polarization $P_{\mathrm{b}}$, background fractions $f_{i}$ with asymmetries $A_{i}$ and finite kinematic acceptance $K$. These corrections are summarized in Table 1 and explained in detail in our forthcoming publication [9]. The first line lists the cumulative $A_{\text {beam }}$ corrections discussed above, scaled by $K / P_{\mathrm{b}}$. 
TABLE 1. Corrections to $A_{\text {corr }}$ and systematic errors.

\begin{tabular}{|l|rll|rll|}
\hline Correction (ppb) & \multicolumn{3}{|c|}{ Helium } & \multicolumn{3}{|c|}{ Hydrogen } \\
\hline \hline Beam Asyms. & 183 & \pm & 59 & -10 & \pm & 17 \\
Target windows & 113 & \pm & 32 & 7 & \pm & 19 \\
Helium QE & 12 & \pm & 20 & & - & \\
Rescatter & 20 & \pm & 15 & 2 & \pm & 4 \\
Nonlinearity & 0 & \pm & 58 & 0 & \pm & 15 \\
\hline \hline Scale Factor & \multicolumn{3}{|c|}{ Helium } \\
\hline Acceptance $K$ & 1.000 & \pm & 0.001 & 0.979 & \pm & 0.002 \\
$Q^{2}$ Scale & 1.000 & \pm & 0.009 & 1.000 & \pm & 0.017 \\
Polarization $P_{\mathrm{b}}$ & 0.844 & \pm & 0.008 & 0.871 & \pm & 0.009 \\
\hline
\end{tabular}

\section{RESULTS FOR $G_{E}^{s}$ AND $G_{M}^{s}$}

The theoretical predictions $A_{\mathrm{NS}}^{\mathrm{He}}$ and $A_{\mathrm{NS}}^{\mathrm{H}}$ with $G^{s}=0$ were estimated using the formalism in [7] and described in our publications $[12,11]$.

Assuming a pure isoscalar $0^{+} \rightarrow 0^{+}$transition, $A_{\mathrm{NS}}^{\mathrm{He}}$ is completely independent of nuclear structure and determined purely by electroweak parameters. D-state and isospin admixtures and meson-exchange currents are negligible at the level of the experimental fractional accuracy of $\sim 3 \%$ [16]. For our kinematics $\left(E_{b}=2.75 \mathrm{GeV},\left\langle Q^{2}\right\rangle=\right.$ $0.077 \mathrm{GeV}^{2}$ ) we obtain $A_{\mathrm{NS}}^{\mathrm{He}}=+6.37 \mathrm{ppm}$.

Electromagnetic form factors from a phenomenological fit to the world data at low $Q^{2}$ [17] were used to calculate $A_{\mathrm{NS}}^{\mathrm{H}}$, with uncertainties governed by data near $Q^{2} \sim 0.1 \mathrm{GeV}^{2}$. The value used for $G_{E}^{\gamma n}=0.037$, with a $10 \%$ relative uncertainty based on new data from the BLAST experiment [18]. For our kinematics $\left(E_{b}=3.18 \mathrm{GeV}\right.$, $\left\langle Q^{2}\right\rangle=0.109 \mathrm{GeV}^{2}$ ) we obtain $A_{\mathrm{NS}}^{\mathrm{H}}=-1.66 \pm 0.05 \mathrm{ppm}$. This includes a contribution from the axial form factor $G_{A}^{Z}$, and associated radiative corrections [19], of $-0.037 \pm 0.018 \mathrm{ppm}$.

Comparing our results to the theoretical expectations, we extract $G_{E}^{s}=0.002 \pm$ $0.014 \pm 0.007$ at $Q^{2}=0.077 \mathrm{GeV}^{2}$ and $G_{E}^{s}+0.09 G_{M}^{s}=0.007 \pm 0.011 \pm 0.004 \pm 0.005$ (FF) at $Q^{2}=0.109 \mathrm{GeV}^{2}$, where the uncertainties in the nucleon electromagnetic form factors govern the last error. Figure 1 displays the combined result for these and our previous measurements $[11,12]$, taken with $\left\langle Q^{2}\right\rangle$ between $0.077-0.109 \mathrm{GeV}^{2}$. The requisite small extrapolation to a common $Q^{2}=0.1 \mathrm{GeV}^{2}$ was made assuming that $G_{E}^{s} \propto Q^{2}$ and that $G_{M}^{S}$ is constant. The values $G_{E}^{S}=-0.005 \pm 0.019$ and $G_{M}^{S}=0.18 \pm 0.27$ (correlation coefficient $=-0.87$ ) are obtained. The results are quite insensitive to variations in $G_{A}^{Z}$, as evidenced by the negligible change induced by an alternate fit similar to that in [20], where $G_{A}^{Z}$ is constrained by other $A_{\mathrm{PV}}$ data.

Figure 1 also displays predictions from selected theoretical models [21, 22, 23, 24, $25,26]$. Those that predict little strange quark dynamics in the vector form factors are favored [25, 26]. Other measurements [13, 14, 15] that had suggested non-zero strangeness effects are consistent, within quoted uncertainties, with our results at $Q^{2}=$ 


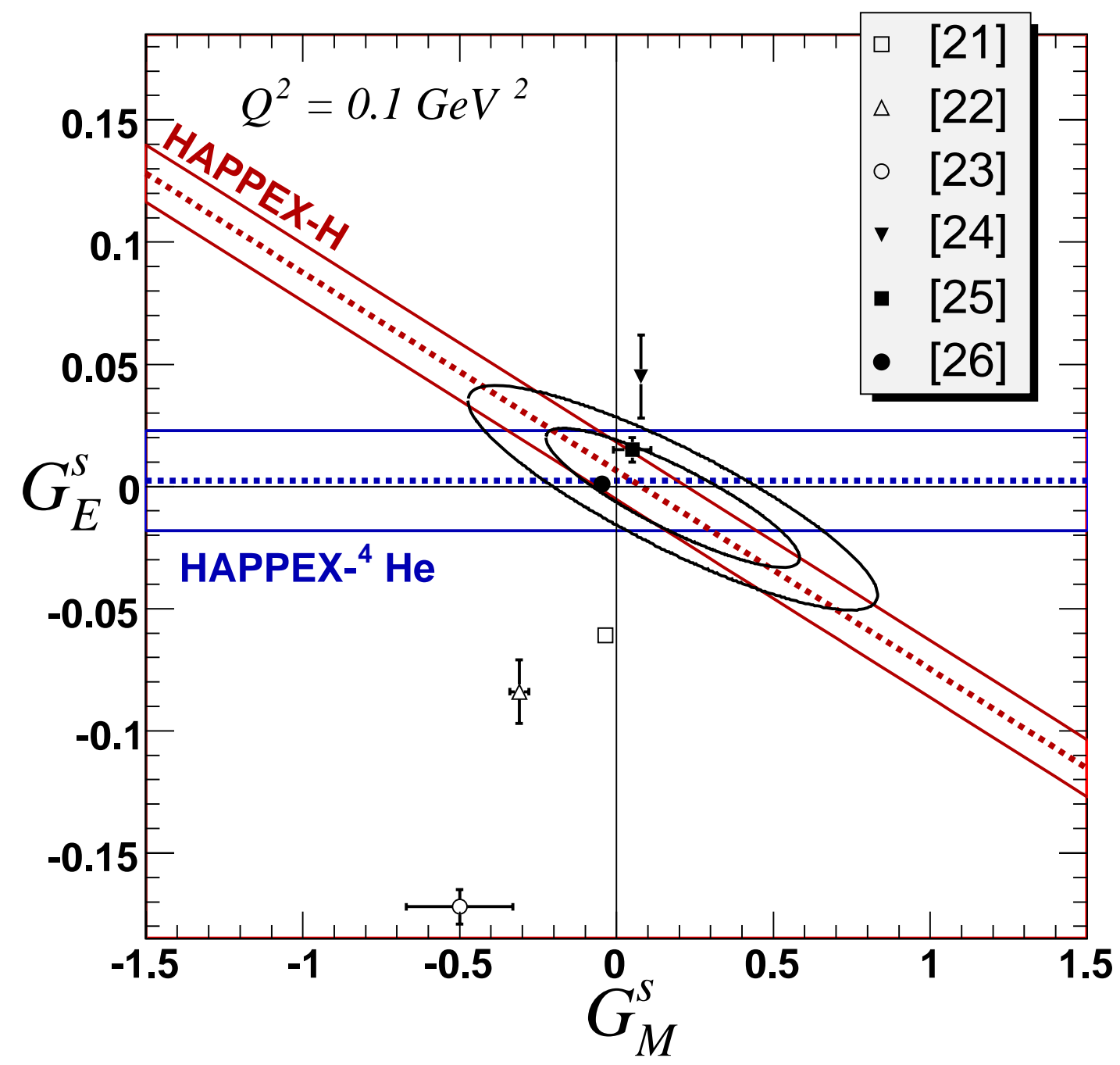

FIGURE 1. 68 and 95\% C.L. constraints in the $G_{E}^{s}-G_{M}^{s}$ plane from data from this apparatus $([11,12]$ and this contribution). Various theoretical predictions are plotted. The 1- $\sigma$ bands (a quadrature sum of statistical and systematic errors) and central values from the new results alone are also shown.

$0.1 \mathrm{GeV}^{2}$. Due to the improved statistical precision and lower $G_{A}^{Z}$ sensitivity of our result, adding these earlier published measurements does not alter our conclusions.

\section{SUMMARY AND OUTLOOK}

In summary, we have reported the most precise constraints on the strange form factors at $Q^{2} \sim 0.1 \mathrm{GeV}^{2}$. The results are consistent with other parity experiments $[13,14,15]$ and suggest a very small contribution of strange quarks at low $Q^{2}$. The world-wide parity violation program is providing a detailed picture of the $s \bar{s}$ contributions to nucleon structure at various distance scales and will place important constraints on theoretical 
models of non-perturbative QCD.

\section{REFERENCES}

1. R. D. McKeown, Phys. Lett. B 219 (1989) 140.

2. E. J. Beise and R. D. McKeown, Comments Nucl. Part. Phys. 20 (1991) 105.

3. D. H. Beck, Phys. Rev. D 39 (1989) 3248.

4. K.S. Kumar and P.A. Souder, Prog. Part. Nucl. Phys. 45 (2000) S333.

5. D.H. Beck and R.D. McKeown, Ann. Rev. Nucl. Part. Sci. 51 (2001) 189.

6. D.H. Beck and B.R. Holstein, Int. J. Mod. Phys. E10 (2000) 1.

7. M.J. Musolf et al., Phys. Rep. 239 (1994) 1.

8. B. Kubis and R. Lewis, Phys. Rev. 74, 015204 (2006).

9. A. Acha, et al., nucl-ex/0609002.

10. K. A. Aniol et al., Phys. Rev. C 69, 065501 (2004).

11. K.A. Aniol et al., Phys. Rev. Lett. 96, 022003 (2006).

12. K.A. Aniol et al., Phys. Lett. B 635, 275 (2006).

13. D.T. Spayde et al., Phys. Lett. B 583, 79 (2004).

14. F.E. Maas et al., Phys. Rev. Lett. 94, 152001 (2005).

15. D.S. Armstrong et al., Phys. Rev. Lett. 95, 092001 (2005).

16. M.J. Musolf, R. Schiavilla and T.W. Donnelly, Phys. Rev. C 50, 2173 (1994); S. Ramavataram, E. Hadjimichael and T.W. Donnelly, Phys. Rev. C 50, 1175 (1994); M.J. Musolf and T.W. Donnelly, Phys. Lett. B 318, 263 (1993).

17. J. Friedrich and Th. Walcher, Eur. Phys. J. A 17, 607 (2003).

18. V. Ziskin, PhD. thesis, MIT, 2005.

19. S.-L. Zhu et al., Phys. Rev. D 62, 033008 (2000).

20. R. Young et al., Phys. Rev. Lett. 97, 102002 (2006).

21. N.W. Park and H. Weigel, Nucl. Phys. A 541, 453 (1992).

22. H.-W. Hammer, U.-G. Meissner, and D. Drechsel, Phys. Lett. B 367, 323 (1996).

23. H.-W. Hammer and M.J. Ramsey-Musolf, Phys. Rev. C 60, 045204 (1999); ibid 60, 045205 (1999); erratum ibid 62, 049902 (2000); 63, 049903 (2000).

24. A. Silva, H.-C. Kim, and K. Goeke, Phys. Rev. D 65, 014016 (2002), Erratum-ibid. D 66, 039902 (2002).

25. R. Lewis, W. Wilcox, and R.M. Woloshyn, Phys. Rev. D 67, 013003 (2003).

26. D.B. Leinweber et al., Phys. Rev. Lett. 94, 212001 (2005); D.B. Leinweber et al., Phys. Rev. Lett. 97, 022001 (2006). 